\title{
EMNLP 2021
}

The Third Workshop on New Frontiers in Summarization (NewSum 2021)

\section{Workshop Proceedings}

November 10, 2021 
C2021 The Association for Computational Linguistics

Order copies of this and other ACL proceedings from:

Association for Computational Linguistics (ACL)

209 N. Eighth Street

Stroudsburg, PA 18360

USA

Tel: +1-570-476-8006

Fax: +1-570-476-0860

acleaclweb.org

ISBN 978-1-955917-04-9 


\section{Message from the Workshop Chairs}

Developing intelligent systems which can produce concise, fluent, and accurate summaries has been a long-standing goal in natural language processing. The aim of this workshop is to provide a research forum for cross-fertilization of ideas towards this goal. We seek to bring together researchers from a diverse range of fields (e.g., summarization, language generation, cognitive and psycholinguistics) for discussions on key issues related to automatic summarization. This includes discussion on novel paradigms/frameworks, multilingual and cross-lingual setups, shared tasks of interest, information integration and presentation, new evaluation protocols, applied research and applications, and possible future research foci. The workshop aims to pave the way towards building a cohesive research community, accelerating knowledge diffusion, developing new tools, datasets, and resources that are in line with the needs of academia, industry, and government. This is the third edition of the workshop, following our previous workshops at EMNLP 2017 and EMNLP 2019. The workshop received 18 long paper submissions, of which 9 were accepted, and 14 short paper submissions, of which 7 were accepted. This resulted in an overall acceptance rate of 50\%. We are pleased to have three invited speakers at this year's workshop: Asli Celikyilmaz (Facebook AI Research), Sebastian Gehrmann (Google Research), and Shashi Narayan (Google Research). Together, they cover a broad spectrum of work in summarization and adjacent areas. We would like to thank our invited speakers, as well as our programme committee members and workshop participants.

Giuseppe Carenini, Jackie Chi Kit Cheung, Yue Dong, Fei Liu, Lu Wang 



\section{Organizing Committee}

Giuseppe Carenini (University of British Columbia, Canada)

Jackie Chi Kit Cheung (McGill University, Canada)

Yue Dong (McGill University \& MILA, Canada)

Fei Liu (University of Central Florida, USA)

Lu Wang (University of Michigan, USA)

\section{Invited Speakers}

Shashi Narayan (Google)

Asli Celikyilmaz (Facebook AI Research)

Sebastian Gehrmann (Google)

\section{Technical Committee}

Enamul Hoque (York University)

Jiacheng $\mathrm{Xu}$ (The University of Texas at Austin)

Rui Zhang (Penn State University)

Hou Pong Chan (University of Macau)

Yuntian Deng (Harvard University)

Kristjan Arumae (Amazon)

Xiaojun Wan (Peking University)

Chris Kedzie (Rasa Technologies Inc.)

Naoaki Okazaki (Tokyo Institute of Technology)

Manabu Okumura (Tokyo Institute of Technology)

Yang Liu (Microsoft)

Tadashi Nomoto (National Institute of Japanese Literature)

Linzi Xing (University of British Columbia)

Ari Rappoport (Hebrew University)

Felice Dell'Orletta (Istituto di Linguistica Computazionale "A. Zampolli" (CNR), Pisa, Italy)

Margot Mieskes (University of Applied Sciences Darmstadt, Germany)

Rodrigo Souza Wilkens (University of Essex)

Maxime Peyrard (EPFL)

Benoit Favre (Aix-Marseille University LIS/CNRS)

Tobias Falke (Amazon)

Thiago Alexandre Salgueiro Pardo (University of São Paulo)

Jessica Ouyang (University of Texas at Dallas)

Wencan Luo (Google)

Florian Boudin (Université de Nantes - France)

Juan-Manuel Torres-Moreno (LIA Avignon Université)

Michael Elhadad (Ben Gurion University)

Esaú Villatoro Tello (Universidad Autónoma Metropolitana Unidad Cuajimalpa, México)

Yuning Mao (University of Illinois at Urbana-Champaign)

Wen Xiao (University of British Columbia)

Xinyu Hua (Northeastern University)

Patrick Huber (University of British Columbia)

Abram Handler (University of Colorado)

Wojciech Kryściński (Salesforce Research) 
Alexander Fabbri (Yale University)

Greg Durrett (UT Austin)

Yang Gao (Royal Holloway, University of London, UK)

Ramakanth Pasunuru (UNC Chapel Hill)

Ido Dagan (Bar-Ilan University) 


\section{Table of Contents}

Sentence-level Planning for Especially Abstractive Summarization

Andreas Marfurt and James Henderson . . . . . . . . . . . . . . . . . . . . . . . . . . . . . . .

Template-aware Attention Model for Earnings Call Report Generation

Yangchen Huang, Prashant K. Dhingra and Seyed Danial Mohseni Taheri ................ 15

Knowledge and Keywords Augmented Abstractive Sentence Summarization

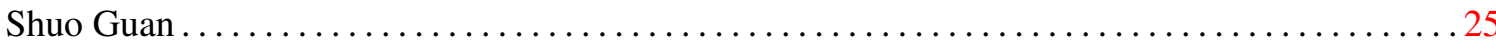

Rewards with Negative Examples for Reinforced Topic-Focused Abstractive Summarization

Khalil Mrini, Can Liu and Markus Dreyer...................................... 33

A Novel Wikipedia based Dataset for Monolingual and Cross-Lingual Summarization

Mehwish Fatima and Michael Strube ....................................... 39

Evaluation of Summarization Systems across Gender, Age, and Race

Anna Jørgensen and Anders Søgaard ..................................... 51

Evaluation of Abstractive Summarisation Models with Machine Translation in Deliberative Processes

Miguel Arana-Catania, Rob Procter, Yulan He and Maria Liakata ....................... 57

Capturing Speaker Incorrectness: Speaker-Focused Post-Correction for Abstractive Dialogue Summarization

Dongyub Lee, Jungwoo Lim, Taesun Whang, chanhee lee, Seungwoo Cho, Mingun Park and

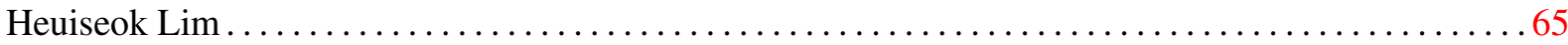

Measuring Similarity of Opinion-bearing Sentences

Wenyi Tay, Xiuzhen Zhang, Stephen Wan and Sarvnaz Karimi ...................... 74

EASE: Extractive-Abstractive Summarization End-to-End using the Information Bottleneck Principle

Haoran Li, Arash Einolghozati, Srinivasan Iyer, Bhargavi Paranjape, Yashar Mehdad, Sonal Gupta

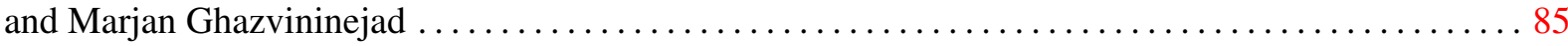

Context or No Context? A preliminary exploration of human-in-the-loop approach for Incremental Temporal Summarization in meetings

Nicole Beckage, Shachi H Kumar, Saurav Sahay and Ramesh Manuvinakurike ............ 96

Are We Summarizing the Right Way? A Survey of Dialogue Summarization Data Sets

Don Tuggener, Margot Mieskes, Jan Deriu and Mark Cieliebak ..................... 107

Modeling Endorsement for Multi-Document Abstractive Summarization

Logan Lebanoff, Bingqing Wang, Zhe Feng and Fei Liu ......................... 119

SUBSUME: A Dataset for Subjective Summary Extraction from Wikipedia Documents

Nishant Yadav, Matteo Brucato, Anna Fariha, Oscar Youngquist, Julian Killingback, Alexandra

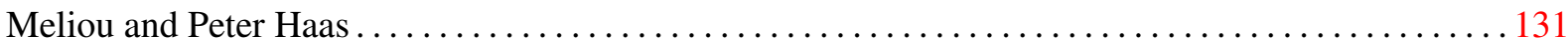

TLDR9+: A Large Scale Resource for Extreme Summarization of Social Media Posts

Sajad Sotudeh, Hanieh Deilamsalehy, Franck Dernoncourt and Nazli Goharian 142 
A New Dataset and Efficient Baselines for Document-level Text Simplification in German

Annette Rios, Nicolas Spring, Tannon Kew, Marek Kostrzewa, Andreas Säuberli, Mathias Müller

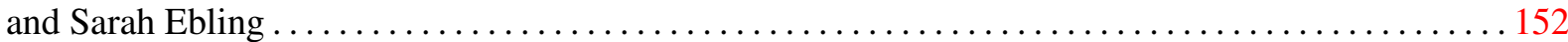




\title{
Conference Program
}

\author{
9:00-10:30 Morning Session I \\ 9:00-9:10 Open remarks \\ NewSum Organizers \\ 9:10-10:00 Keynote I \\ Sashi Narayan (Google) \\ 10:00-10:10 Sentence-level Planning for Especially Abstractive Summarization \\ Andreas Marfurt and James Henderson \\ 10:10-10:20 Template-aware Attention Model for Earnings Call Report Generation \\ Yangchen Huang, Prashant K. Dhingra and Seyed Danial Mohseni Taheri \\ 10:20-10:25 Knowledge and Keywords Augmented Abstractive Sentence Summarization \\ Shuo Guan \\ 10:25-10:30 Rewards with Negative Examples for Reinforced Topic-Focused Abstractive Sum- \\ marization \\ Khalil Mrini, Can Liu and Markus Dreyer
}

\section{0:30-11:00 Coffee break I}

\section{1:00-12:00 Morning session II}

11:00-11:50 Keynote II

Sebastian Gehrmann (Google)

11:50-12:00 A Novel Wikipedia based Dataset for Monolingual and Cross-Lingual Summarization

Mehwish Fatima and Michael Strube

\section{2:00-13:00 Lunch break}




\section{November 10 (continued)}

\section{3:00-14:30 Afternoon session I}

13:00-13:50 Keynote III

Asli Celikyilmaz (Facebook AI Research)

13:50-13:55 Evaluation of Summarization Systems across Gender, Age, and Race

Anna Jørgensen and Anders Søgaard

13:55-14:00 Evaluation of Abstractive Summarisation Models with Machine Translation in Deliberative Processes

Miguel Arana-Catania, Rob Procter, Yulan He and Maria Liakata

14:00-14:10 Capturing Speaker Incorrectness: Speaker-Focused Post-Correction for Abstractive Dialogue Summarization

Dongyub Lee, Jungwoo Lim, Taesun Whang, chanhee lee, Seungwoo Cho, Mingun Park and Heuiseok Lim

14:10-14:20 Measuring Similarity of Opinion-bearing Sentences

Wenyi Tay, Xiuzhen Zhang, Stephen Wan and Sarvnaz Karimi

14:20-14:30 EASE: Extractive-Abstractive Summarization End-to-End using the Information Bottleneck Principle

Haoran Li, Arash Einolghozati, Srinivasan Iyer, Bhargavi Paranjape, Yashar Mehdad, Sonal Gupta and Marjan Ghazvininejad

\section{4:30-15:00 Coffee break}

\section{5:00-16:15 Afternoon session II}

15:00-15:10 Context or No Context? A preliminary exploration of human-in-the-loop approach for Incremental Temporal Summarization in meetings

Nicole Beckage, Shachi H Kumar, Saurav Sahay and Ramesh Manuvinakurike

15:10-15:20 Are We Summarizing the Right Way? A Survey of Dialogue Summarization Data Sets

Don Tuggener, Margot Mieskes, Jan Deriu and Mark Cieliebak

15:20-15:30 Modeling Endorsement for Multi-Document Abstractive Summarization Logan Lebanoff, Bingqing Wang, Zhe Feng and Fei Liu 


\section{November 10 (continued)}

15:30-15:35 SUBSUME: A Dataset for Subjective Summary Extraction from Wikipedia Documents

Nishant Yadav, Matteo Brucato, Anna Fariha, Oscar Youngquist, Julian Killingback, Alexandra Meliou and Peter Haas

\section{5:35-16:15 EMNLP finding papers - Summarization}

\section{6:15-16:45 Coffee break}

\section{6:45-18:00 Afternoon session III}

16:45-16:55 TLDR9+: A Large Scale Resource for Extreme Summarization of Social Media Posts

Sajad Sotudeh, Hanieh Deilamsalehy, Franck Dernoncourt and Nazli Goharian

16:55-17:00 A New Dataset and Efficient Baselines for Document-level Text Simplification in German

Annette Rios, Nicolas Spring, Tannon Kew, Marek Kostrzewa, Andreas Säuberli, Mathias Müller and Sarah Ebling

\section{7:00-18:00 Mentoring Program}


